\title{
Becker muscular dystrophy patient with a large intragenic dystrophin deletion: implications for functional minigenes and gene therapy
}

\author{
Donald R Love, Tracey J Flint, Sally A Genet, Helen R Middleton-Price, Kay E Davies
}

\begin{abstract}
The genetic defects responsible for the allelic disorders of BMD and the more severe DMD have been shown to be mutations within the dystrophin gene, which encodes a $14 \mathrm{~kb}$ transcript. We describe here a BMD patient who belongs to a small class of subjects with large in frame deletions of the dystrophin gene that remove apparently dispensable coding sequence, thereby producing functional truncated dystrophin. The in vitro reconstruction of these deletion derivatives of full length dystrophin transcripts should enable higher efficiency transfection of human muscle or murine germline cells using retroviral based vectors, compared with the full length transcript. This capability offers a means of examining retroviral mediated transfer as a potential therapeutic strategy in severely affected DMD patients.
\end{abstract}

The majority of mutations $(\sim 65 \%)$ in Duchenne and Becker muscular dystrophies (DMD, BMD) are intragenic deletions; approximately $10 \%$ of patients have duplications. ${ }^{12}$ The severity of the clinical phenotype does not correlate with the location and extent of deletion, but does appear to correlate with the effect that the mutation has on the translational reading frame of the dystrophin transcript. ${ }^{134}$ In the case of BMD, the reading frame is maintained and,

Molecular Genetics Group, Institute of Molecular Medicine, John Radcliffe Hospital, Headington, Oxford OX3 9DU.

D R Love, T J Flint, K E Davies

Mothercare Department of Paediatric Genetics, Institute of Child Health, University of London, 30 Guilford Street, London WC1N 1EH.

S A Genet, H R Middleton-Price

Correspondence to Dr Davies.

Received for publication 8 April 1991.

Revised version accepted for publication 22 May 1991. in those patients for whom dystrophin has been analysed by Western blotting, an altered size dystrophin is produced. These naturally occurring deletion derivatives of dystrophin in BMD patients provide a resource for mapping functionally important domains of this large $(427 \mathrm{kd})^{5}$ muscle protein and for permitting the construction of functional minigenes.

Analysis of the nucleotide sequence of the dystrophin transcript ${ }^{56}$ together with immunohistochemistry of muscle sections ${ }^{79}$ and electronmicrographic studies of purified dystrophin ${ }^{10}$ have led to a model of dystrophin structure and function. The protein is proposed to consist of four domains: an Nterminal domain (240 amino acids) with putative actin binding function ${ }^{11}$; a rod shaped domain formed by 24 repeat sequences that are predicted to adopt a triple helical conformation with hinge regions $^{1213}$; a cysteine rich domain homologous to the entire C-terminal of Dictyostelium discoideum $\alpha$-actinin $^{6}$; and a 420 amino acid carboxyl-terminal region that is highly conserved in man and chicken ${ }^{14}$ and is homologous to another high molecular weight muscle protein of unknown function..$^{15}$ The latter domain is thought to mediate interaction with a membrane protein complex. ${ }^{16}$ Dystrophin has been shown to self associate in a manner similar to spectrin and $\alpha$-actinin such that it may form a flexible subsarcolemmal network for maintaining membrane integrity, or the spatial configuration of integral membrane proteins during normal contraction induced stress. ${ }^{7131617}$

Here we present Southern hybridisation analysis of a BMD patient with a large deletion of the dystrophin gene within the rod shaped domain. A comparison of this patient's deletion together with those of other BMD patients reported in the literature is discussed with a view to elucidating the minimal length of coding region necessary to give rise to a functional, albeit truncated, dystrophin. The implications for using in vitro constructed minigenes in therapeutic strategies for DMD patients is discussed. 


\section{Patients and methods}

The proband (patient 2389) presented at 21 years of age with proximal weakness of both legs that had become evident three years previously. This weakness was most obvious on climbing stairs and rising from a squat position. EMG investigations suggested an active myopathy and, together with a raised serum creatine kinase level (no data recorded), a diagnosis of $\mathrm{BMD}$ was made. The proband is now 26 years of age and continues to work as a panel beater; however, his maternal uncle, aged 44 years, is mainly confined to a wheelchair. The patient's sisters (see pedigree, fig 1) had serum creatine kinase levels of $1566 \mathrm{U} / 1$ (II 1 ), $204 \mathrm{U} / 1$ (II.2), and $112 \mathrm{U} / 1$ (II.3) (normal range 25 to 170 $\mathrm{U} / \mathrm{l})$. The sister with the highest level of serum
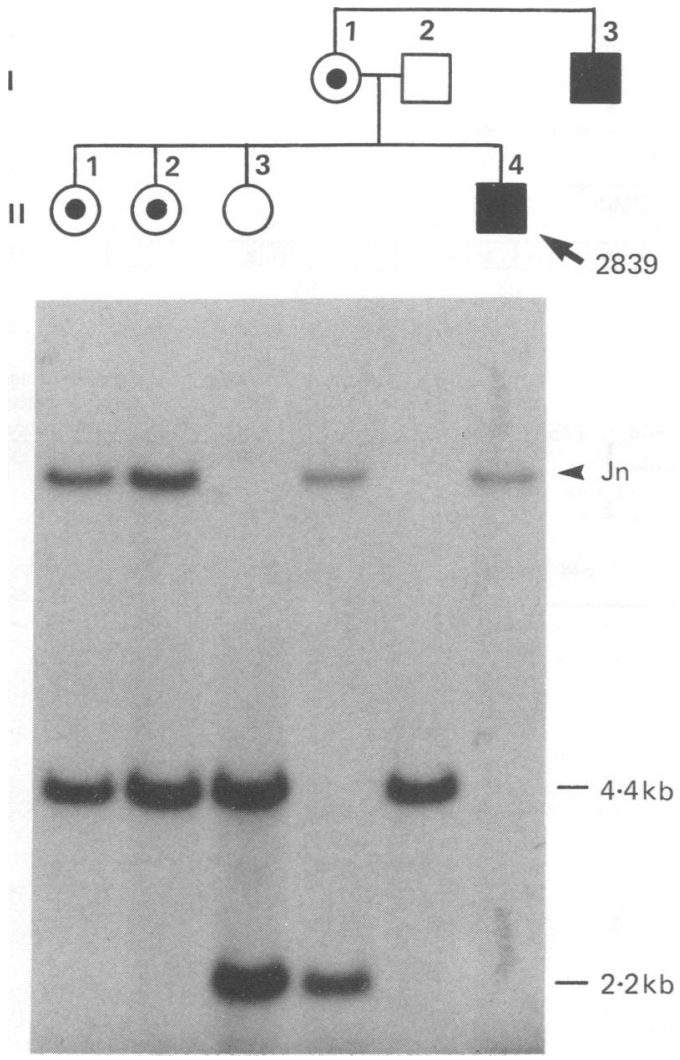

Figure 1 Pedigree and Southern analysis of patient 2839 and family members. Upper panel shows pedigree: $\odot=$ carrier female, $\mathbf{\square}=$ affected male. Lower panel shows pERT87-8 hybridisation with BstXI digested DNA in which the lanes contain DNA of patient 2839 and family members as indicated in the upper panel. The lengths of the alleles detected by $p E R T 87-8$, together with the junction fragment $\left(\mathfrak{f}_{n}\right)$ detected in carrier females and the affected male (patient 2839), are shown. creatine kinase is phenotypically normal but is only 20 years of age.

DNA was isolated from blood lymphocytes by the method described by Jeanpierre ${ }^{18}$ and digested with restriction endonucleases according to the manufacturers' instructions. Digested DNA was electrophoresed in $\mathbf{0 . 8 \%}$ agarose gels, capillary blotted to Hybond $\mathbf{N}$ or Hybond $\mathbf{N}+$ membranes (Amersham), and hybridised with randomly primed ${ }^{32} \mathrm{P}$ labelled probes as described previously. ${ }^{19}$ The DNA probes used were genomic probe pERT87-8 (DXS164, supplied by L M Kunkel), which detects a BstXI polymorphism, ${ }^{20}$ genomic probe P20 (DXS169, supplied by $\mathrm{G}$ van Ommen), ${ }^{21}$ and dystrophin $\mathrm{cDNAs}^{2224}$ (shown in fig 2).

Oligonucleotides flanking dystrophin exon $45^{125}$ were used to amplify genomic sequence by PCR from the proband's DNA following the protocol of Chamberlain et al. ${ }^{25}$

\section{Results}

The family pedigree of patient 2839 is shown in fig 1 , together with the hybridisation analysis of Bst XI digested DNA using the dystrophin genomic probe pERT 87-8 (DXS164). The data indicate that the BMD mutation is segregating with an altered size Bst XI fragment. Preliminary hybridisation analysis of this patient's DNA indicated that he carried a deletion within the dystrophin gene. The location and extent of the deletion were determined by hybridising cDNAs covering nucleotides 1418 to 7880 with HindIII and PstI digested 2839 DNA. The hybridisation analysis was compared with that of patient 324 who has been reported previously with a large intragenic deletion of $5106 \mathrm{bp}$ from exon 17 to 48 , inclusive. ${ }^{26}$

The $5^{\prime}$ deletion endpoint of patient 2839 occurs between exons 13 and 14 (fig $3 \mathrm{~A}$ ). This suggests that the altered size $B s t \mathrm{XI}$ fragment detected by pERT87-8 is a junction fragment because Cf16 maps within the DXS164 locus (fig 2) The $3^{\prime}$ deletion endpoint is located within the P20 intron, which is bounded by exons 44 and 45 . The hybridisation of HindIII and PstI digested 2839 DNA with Cf23a and $\mathrm{Cf} 56 \mathrm{~b}$ (wholly contained within Cf23a), respectively, indicated the absence of exons upstream of exon 45; PCR analysis of 2839 genomic DNA using oligonucleotides flanking exon 45 showed that this exon is intact (data not shown). The precise location of the $3^{\prime}$ endpoint within the P20 intron is not known; however, this endpoint is $5^{\prime}$ to the genomic region containing the P20 cosmid because both HindIII fragments detected by P20 are present in patient 2839 (fig 3B). The full deletion of this patient with respect to the map of the dystrophin gene is shown in fig 2. 


\section{Discussion}

Patient 2839 has an intragenic deletion of $44 \%$ of the coding region of dystrophin that is predicted not to affect the translational reading frame, and his phenotype is mild. These data suggest that a large portion of the rod shaped domain of dystrophin is dispensable without adversely affecting the patient's muscle function. In this regard, patient 2389 is similar to our earlier description of BMD patient 324 who has a deletion of $46 \%$ of dystrophin. ${ }^{26}$ The latter patient's muscle shows a truncated dystrophin of $220 \mathrm{kd}$ by Western analysis and continuous membrane staining for dystrophin by immunohistochemistry.

Few BMD patients have been described with large intragenic deletions ${ }^{12627}$ and, of these, only one has been analysed to determine dystrophin size and localisation. ${ }^{26}$ The data (fig 2 ) indicate that $43 \%$ to
$63 \%$ of the rod shaped domain appears dispensable although there is variation of clinical severity within this deletion range, at least in terms of the age at which patients become wheelchair bound (fig 2). In particular, patient 324, who has the largest intragenic deletion reported so far, was able to walk with the aid of a stick at 61 years of age, and his affected nephew (1353) is a weight trainer at 25 years of age. ${ }^{26}$ The deletion range shown in fig 2 has implications for gene therapy of DMD patients based on retrovirus mediated transfection of muscle cells. The cloning of the coding region of a deleted dystrophin of $5.9 \mathrm{~kb}$ in length (patient 324 ) would permit relatively high efficiency infection of cells by retroviral recombinants ${ }^{28}$ compared with the cloning of a full length transcript (minimum length of $11 \mathrm{~kb}$ ). Retrovirus minigene recombinants offer a means of examining in vivo complementation of mutations that
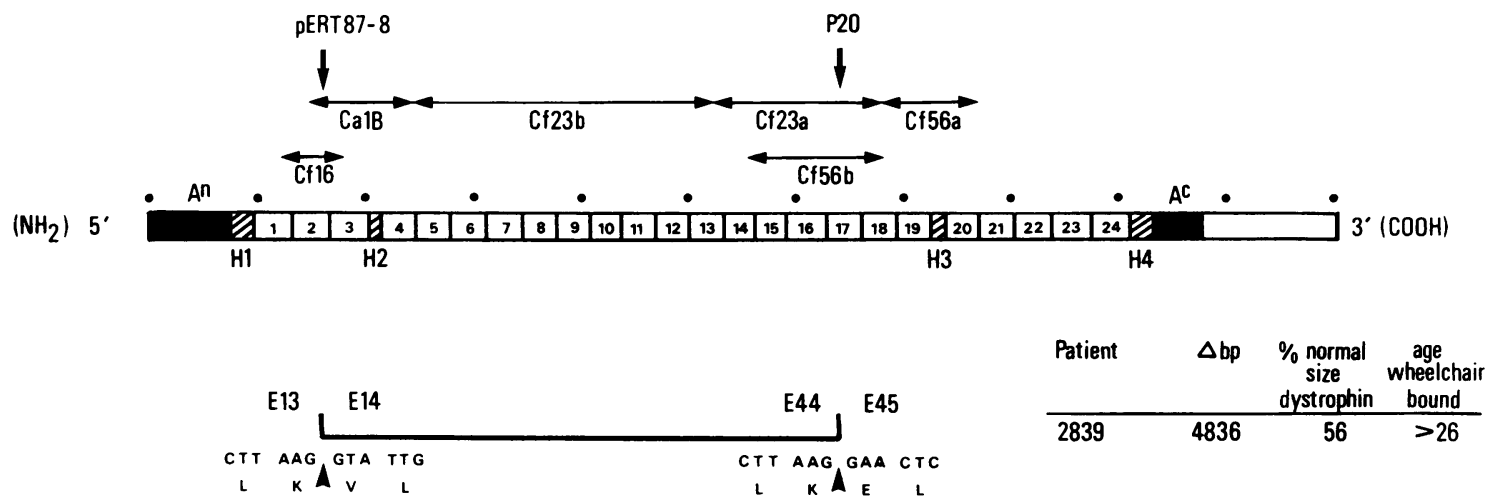



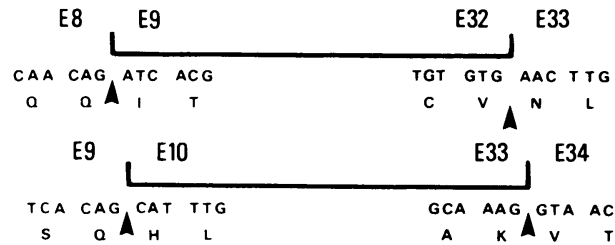

Hodgson et al $^{27} \quad 3687 \quad 67 \quad>16$ no. 44230

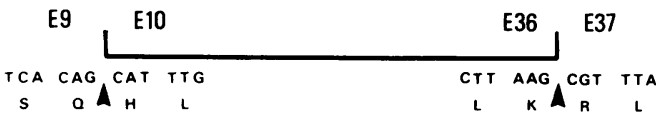

Figure 2 Location and extent of large intragenic deletions in the dystrophin gene of BMD patients. The four domains of dystrophin are represented by: filled boxes, designated $A^{n}$ and $A^{c}$ (regions of homology with amino-and carboxyl-terminal domains of $\alpha$-actinin, respectively); open box is the putative membrane binding domain; the rod shaped domain is divided into 24 repeats with the hatched boxes indicating putative hinge regions $(H 1-H 4) .^{12}$ The location of dystrophin cDNAs (horizontal arrows) and genomic probes (vertical arrows) used in the Southern hybridisation analysis of patients 2839 and 324 are indicated above the domain map and the dots indicate spacings of $1 \mathrm{~kb}$. The patient deletions are indicated below the domain map. Exon boundaries (indicated by arrow heads) defining these deletions are shown by exon number and dystrophin nucleotide and amino acid sequences. Patients still ambulatory are indicated by ' $>$ (age at last clinical examination)'. 


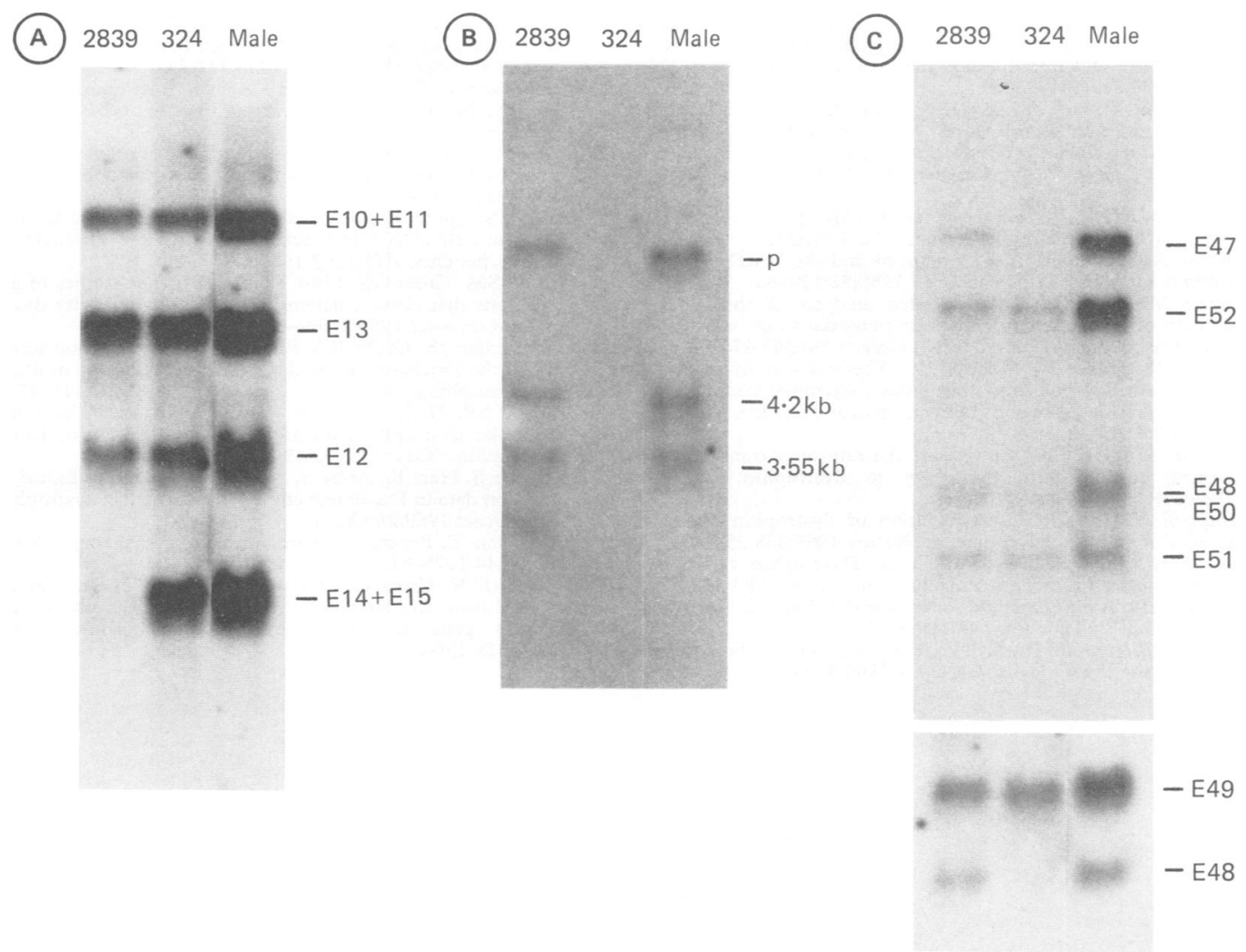

Figure 3 Southern analysis of BMD patients 2839 and 324 using dystrophin gene probes. DNA was digested with HindIII and probed with dystrophin cDNAs Cf16 (A) and Cf56a (C), and the genomic probe P20 (B). Dystrophin exon numbers are indicated alongside appropriate panels and $p$ denotes partial digestion products.

affect translation of endogenous dystrophin genes, for example, in DMD patients and the $m d x$ mouse, which is a rodent model of DMD. ${ }^{29}$

At a functional level, the model described by Koenig and Kunkel $^{13}$ that proposes a flexible network of dystrophin molecules dependent on hinges within the rod domain may require modification. Fig 2 shows that all the BMD patient deletions remove hinge 2 , but retain the region of the rod domain downstream of hinge 3. Electronmicrographic analysis of truncated dystrophin molecules in vitro and sarcolemmal distribution in vivo should aid in determining those structural domains that are important in intermolecular associations. These studies should also provide means of evaluating dystrophin deletion derivatives constructed in vitro with regard to their suitability for gene therapy.

We thank Helen Blaber for typing this manuscript and acknowledge the financial support of the Medical Research Council, the Muscular Dystrophy
Group of Great Britain and Northern Ireland, and the Muscular Dystrophy Association of America.

1 Koenig M, Beggs AH, Moyer M, et al. The molecular basis for Duchenne versus Becker muscular dystrophy: correlation of severity with type of deletion. Am $\mathcal{F}$ Hum Genet 1989;45:498-506.

2 Den Dunnen JT, Grootscholton PM, Bakker E, et al. Topography of the Duchenne muscular dystrophy gene: FIGEand CDNA analysis of 194 cases reveals 115 deletions and 13 duplications. Am 7 Hum Genet 1989;45:835-47.

3 Monaco AP, Bertelson CJ; Liechti-Gallati S, et al. An explanation for the phenotypic differences between patients bearing partial deletions of the DMD locus. Genomics 1988;2:90-5.

4 Hoffman EP, Fischbeck KH, Brown RH, et al. Characterization of dystrophin in muscle-biopsy specimens from patients with Duchenne's or Becker's muscular dystrophy. $N$ Engl f Med 1988;318:1363-8.

5 Koenig M, Monaco AP, Kunkel LM. The complete sequence of dystrophin predicts a rod-shaped cytoskeletal protein. Cell 1988;53:219-28.

6 Koenig M, Hoffman EP, Bertelson CJ, et al. Complete cloning of the Duchenne muscular dystrophy (DMD) cDNA and preliminary genomic organization of the DMD gene in normal and affected individuals. Cell 1987;50:509-17.

7 Arahata K, Ishiura S, Ishiguro T, et al. Immunostaining of skeletal and cardiac muscle surface membrane with antibody against Duchenne muscular dystrophy peptide. Nature 1988;333:861-3. 
8 Arahata K, Hoffman EP, Kunkel LM, et al. Dystrophin diagnosis: comparison of dystrophin abnormalities by immunofluorescence and immunoblot analyses. Proc Natl Acad $\mathrm{Sc}$ USA 1989;86:7154-8.

9 Miyatake M, Miike T, Zhao J-E, et al. Dystrophin: localization and presumed function. Muscle Nerve 1991;14:113-9.

10 Pons F, Augier N, Heilig R, et al. Isolated dystrophin molecules as seen by electron microscopy. Proc Natl Acad Sci USA 1990;87:7851-5.

11 Hammonds RG Jr. Protein sequence of DMD gene is related to actin-binding domain of $\alpha$-actinin. Cell 1987;51:1.

12 Davison MD, Critchley DR. $\alpha$-actinins and the DMD protein contain spectrin-like repeats. Cell 1988;52:159-60.

13 Koenig ME, Kunkel LM. Detailed analysis of the repeat domain of dystrophin reveals four potential hinge segments that may confer flexibility. F Biol Chem 1990;265:4560-6.

14 Lemaire C, Heilig R, Mandel JL. The chicken dystrophin cDNA: striking conservation of the C-terminal coding and the $3^{\prime}$ untranslated regions between man and chicken. EMBO f 1988;7:4157-62.

15 Love DR, Hill DF, Dickson G, et al. An autosomal transcript in skeletal muscle with homology to dystrophin. Nature 1989;339:55-8.

16 Campbell KP, Dahl SD. Association of dystrophin and an integral membrane glycoprotein. Nature 1989;338:259-62.

17 Tay JSH, Low PS, Lee WL, et al. Dystrophin function: calcium related rather than mechanical Lancet 1989;335:83.

18 Jeanpierre M. A rapid method for the purification of DNA from blood. Nucleic Acids Res 1987;15:9611.

19 Love DR, Bloomfield JF, Kenwrick SJ, et al. Physical mapping distal to the DMD locus. Genomics 1990;8:106-12.
20 Kunkel LM, Hejtmancik JF, Caskey CT, et al. Analysis of deletions in DNA from patients with Becker and Duchenne muscular dystrophy. Nature 1986;322:73-7.

21 Wapenaar MC, Krevits T, Hart KA, et al. A deletion hotspot in the Duchenne muscular dystrophy gene. Genomics 1988; 2:101-8.

22 Forrest SM, Cross GS, Speer A, et al. Preferential deletion of exons in Duchenne and Becker muscular dystrophies. Nature 1987;329:638-40.

23 Cross GS, Speer A, Rosenthal A, et al. Deletions of fetal and adult muscle cDNA in Duchenne and Becker muscular dystrophy patients. $E M B O \mathcal{F} 1987 ; 6: 3277-83$.

24 Forrest SM, Cross GS, Flint T, et al. Further studies of gene deletions that cause Duchenne and Becker muscular dystrophies. Genomics 1988;2:109-14.

25 Chamberlain JS, Gibbs RA, Ranier JE, et al. Deletion screening of the Duchenne muscular dystrophy locus via multiplex DNA amplification. Nucleic Acids Res 1988;16:11141-57.

26 England SB, Nicholson LVB, Johnson MA, et al. Very mild muscular dystrophy associated with the deletion of $46 \%$ of dystrophin. Nature 1990;343:180-2.

27 Hodgson S, Hart K, Abbs S, et al. Correlation of clinical and deletion data in Duchenne and Becker muscular dystrophy. $f$ Med Genet 1989;26:682-93.

28 Friedmann T. Progress towards human gene therapy. Science 1989;244:1275-81.

29 Brockdorff N, Cross GS, Cavanna JS, et al. The mapping of cDNA from the human $\mathrm{X}$-linked Duchenne muscular dystrophy gene to the mouse $X$ chromosome. Nature 1987;328:166-8. 